Aim of the study: This study aimed to compare the efficacy and toxicity of docetaxel combined with cisplatin (DP) and gemcitabine combined with cisplatin (GP) in postoperative chemotherapy after surgery of non-small cell lung cancer (NSCLC).

Material and methods: A total of 92 patients diagnosed with NSCLC after surgery were enrolled, and they were treated with DP (DP group) and GP (GP group). The efficacy and toxicity of the medications were then compared. Results: Approximately 92.4\% (85 out of 92) of the patients received chemotherapy for more than three weeks. In the DP and GP groups, the incidence rates of grade III-IV thrombocytopenia were $24.4 \%$ and $6.38 \%$, respectively, whereas the incidence rates of alopecia were $88.9 \%$ and $25.5 \%$, respectively. The difference between the two groups was statistically significant $(p<0.05)$. Disease-free survival rates in DP group in one and two years were $76.5 \%$ and $50.47 \%$, respectively, whereas in the GP group they were $77.8 \%$ and $49.52 \%$, respectively. No significant difference was observed between the two groups $(p>0.05)$. Conclusions: These results showed similar disease-free survival rates of DP and GP therapies in one and two years after surgery for NSCLC. However, the DP group exhibited higher incidence rates of grade III-IV thrombocytopenia and alopecia than the GP group. Therefore, we should select a specific treatment for each patient according to individual differences.

Key words: NSCLC, postoperative chemotherapy, docetaxel, gemcitabine,cisplatin.

Contemp Oncol (Pozn) 2015; 19 (4): 323-326 DOI: $10.5114 /$ wo.2015.53373

\section{Clinical observation of docetaxel or gemcitabine combined with cisplatin in the chemotherapy after surgery for stage II-III non-small cell lung cancer}

\author{
Qiu-Qiang Chen ${ }^{1}$, Xue-Xian $\mathrm{Ji}^{2}$, Xiao Zhou ${ }^{3}$, Qi-Lin Shi ${ }^{4}$, Huan-Ming $\mathrm{Yu}^{1}$, \\ Heng-Qin Fü, Guo-Ping Ji ${ }^{5}$
}
${ }^{1}$ Department of Thoracic Surgery, the directly affiliated hospital of Huzhou Teachers College, Huzhou, Zhejiang, PR China
2Department of Pathology, People's Hospital of Deqing County, Wukang, Zhejiang, PR China
${ }^{3}$ Department of Respiratory Medicine, the directly affiliated hospital of Huzhou Teachers College, Huzhou, Zhejiang, PR China
${ }^{4}$ Department of Pathology, the directly affiliated hospital of Huzhou Teachers College, Huzhou, Zhejiang, PR China
${ }^{5}$ Department of Respiratory Medicine, People's Hospital of Deqing County, Wukang, Zhejiang, PR China

\section{Introduction}

Lung cancer has become a life-threatening disease with the highest incidence and mortality among cancer types in large and medium-sized cities of China; lung cancer is also recognised as one of the main causes of death in the world [1]. Non-small cell lung cancer (NSCLC) accounts for $80 \%$ to $85 \%$ of lung cancer cases [2], mostly diagnosed at stages II to III. Better efficacy in stage II to III NSCLC is observed in surgery-based chemotherapy or radiation therapy compared with chemotherapy or radiation therapy alone [3-5]. Chemotherapy is an important part of combined therapy for NSCLC and can enhance the survival rate of patients subjected to surgery [6]. Cisplatin-based adjuvant chemotherapy is recommended by the American Society of Clinical Oncology for adjuvant chemotherapy and adjuvant radiation therapy of stages II to IIIA NSCLC published on "Journal of Clinical Oncology" in 2007 [7]. However, standard chemotherapy has not been applied in clinical research and the selection of appropriate chemotherapy remains a challenge. In current clinical studies, docetaxel or gemcitabine combined with cisplatin (DP or GP, respectively) is widely applied, thereby eliciting a definite effect for NSCLC, but only a few retrospective comparisons of efficacy and toxicity between these two chemotherapies have been published. Therefore, we collected data based on GP or DP treatment of NSCLC after surgery in two hospitals in 2004. We observed and analysed whether or not postoperative recurrence rates and toxic effects are possibly reduced. We also determined whether or not the treatment increases long-term survival to provide evidence for personalised treatment of NSCLC.

\section{Material and methods}

\section{Patient selection}

From January 1, 2002 to December 31, 2007, patients with NSCLC who underwent surgery were selected according to the following standards: 1) age < 70 years; 2) PS 0 to 2; 3) expected survival longer than 6 months; 4) no chemotherapy, radiation therapy, or molecular targeted therapy before 
surgery; 5) operation method was pulmonary lobectomy or pneumonectomy plus lymphadenectomy; 6) pathological type was NSCLC; 7) postoperative pathological stage for TNM was II to III; 8) respiratory condition after surgery should be normal or only slight dyspnoea after minor activity; 9) patients were normal in blood routine test, hepatorenal function, and electrocardiogram (ECG); and 10) willing to undergo a follow-up study. This study was conducted in accordance with the Declaration of Helsinki. This study was conducted with approval from the Ethics Committee of the directly affiliated hospital of Huzhou Teachers College. Written informed consent was obtained from all participants.

\section{General data}

Among 112 surgical patients with NSCLC, 5 patients who refused further treatment after surgery and 15 patients who did not finish three cycles of chemotherapy were excluded. The remaining 92 patients were randomised into DP and GP groups. A total of 45 patients aged 32 to 67 years (median age -55 years) in the DP group consisted of 38 males and 7 females, including 27 cases of adenocarcinoma, 17 cases of squamous carcinoma and 1 case of adenosquamous carcinoma. A total of 47 patients aged 31 to 67 years (median age -56 years) in the GP group consisted of 41 males and 6 females, including 29 cases of adenocarcinoma, 17 cases of squamous carcinoma and 1 case of adenosquamous carcinoma. The patients in the 2 groups did not show significant differences in clinical features $(p>0.05)$. None of the cases were subjected to the EGFR test.

\section{Therapeutic method}

DP Group. Docetaxel $75 \mathrm{mg} / \mathrm{m}^{2}$ was added to physiological saline solution $(250 \mathrm{ml})$. The resulting solution was intravenously instilled for $1 \mathrm{~h}$ with ECG monitoring, d1. Cisplatin $\left(75 \mathrm{mg} / \mathrm{m}^{2}\right)$ was intravenously instilled from $1 \mathrm{~d}$ to $3 \mathrm{~d}, \mathrm{~d}_{1-3}$. To reduce body fluid retention at $1 \mathrm{~d}$ before the treatment, the patients were administered with $8 \mathrm{mg}$ of oral dexamethasone twice per day for 3 days. GP Group. Gemcitabine $\left(1250 \mathrm{mg} / \mathrm{m}^{2}\right)$ was diluted in physiological saline solution $(150 \mathrm{ml})$. The resulting solution was intravenously instilled for $0.5 \mathrm{~h}$ in $\mathrm{d}_{1}$, 8. Cisplatin $\left(75 \mathrm{mg} / \mathrm{m}^{2}\right)$ was intravenously instilled from $1 \mathrm{~d}$ to $3 \mathrm{~d}, \mathrm{~d}_{1-3}$. In these two therapies, one cycle covered 21 days and each patient received at least 3 cycles of chemotherapy to evaluate its efficacy and side effects. Tropisetron and other similar medications were routinely administered to stop vomiting during chemotherapy. Blood routine, hepatorenal function and electrolytes were reviewed twice per week during chemotherapy. The patients with $<3.5 \times 10^{9}$ leucocytes/l underwent granulocyte colony-stimulating factor treatment to increase the concentration of leucocytes. The patients who were subjected only to surgery did not receive any chemotherapy.

\section{Follow-up}

Medical records were collected, including patient age, surgery date, and other pertinent data. These records were reviewed in the clinic or followed up by telephone to re- cord the time of recurrence and metastasis. A follow-up session was conducted twice per year until December 31, 2009 with a follow-up rate of $95.5 \%$ ( 5 cases were lost). The median follow-up time was 679 days. The loss of follow-up, the survival cases until the last follow-up or the non-cancer-related death cases were processed as the censored value according to statistical analysis.

\section{Observation of indices}

The patients underwent several examinations before the treatment and every 2 weeks after the treatment, including medical history, physical functioning, blood routine and hepatorenal function. Toxic response was graded according to CTCAE $\vee 3.0$ criteria [8].

The survival rates in 1 and 2 years were counted from the beginning of surgery until the death after the last follow-up or the loss of follow-up.

Disease-free survival was monitored beginning from the last stage of primary lung cancer surgery to the local recurrence of lung cancer or distant metastasis. This factor should be confirmed by computed tomography (CT), magnetic resonance imaging (MRI), electrical capacitance tomography (ECT), positron emission tomography computed tomography (PET-CT) or biopsy.

\section{Statistical analysis}

Statistical analysis was performed in SPSS 10.0 software. $\chi^{2}$ and Kaplan-Meier methods were used to calculate the survival rate. $p<0.05$ was considered as statistically significant.

\section{Results}

\section{Toxic response}

A total of 45 and 47 patients in the DP and GP groups finished more than three cycles of chemotherapy, respectively. No patient died because of a chemotherapy-related toxic effect. The efficacy evaluation was performed more than 3 weeks after the last chemotherapy. The most common toxic responses of the patients in the 2 groups included bone marrow suppression, gastrointestinal reaction and hepatic dysfunction. The incidences of leucocytopenia in the DP and GP groups were $86.7 \%$ and $76.6 \%$, respectively, in which the incidences of grade III-IV leucocytopenia were $20.0 \%$ and $17.0 \%$, respectively, without a significant difference $(p>0.05)$. A statistical difference ( $p<0.05)$ was observed in the incidences of grade III-IV thrombocytopenia and alopecia between the DP group (24.4\% and $88.9 \%$, respectively) and GP group (6.38\% and $25.5 \%$, respectively). The incidences of hepatic dysfunction in DP and GP groups were $26.7 \%$ and $19 \%$, respectively, indicating no significant difference $(p>0.05)$. An allergic response did not occur in either group. There were no statistical differences in haematological toxicity, diarrhoea and rash ( $p>0.05$; Table 1).

\section{Survival rate}

The Kaplan-Meier method was used to calculate the survival rate. The follow-up period ranged from 10 to 42 
months with a median of 22 months. The median OS of all of the patients was 883 days $(95 \%$ Cl 687 to 1027 days) and the overall survival rates in 1,2 and 3 years were $85.5 \%, 64.17 \%$ and $49.61 \%$, respectively. The median disease-free survival (DFS) of all patients was 619 days (95\% $\mathrm{Cl} 411$ to 827 days) and the DFS rates in 1 and 2 years were $75.6 \%$ and $45.0 \%$, respectively.

\section{Survival rate comparison}

The median survival in DP and GP groups was 889 and 880 days, respectively. The survival rates in 1, 2 and 3 years were $93.97 \%, 75.39 \%$ and $50.47 \%$ in the DP group and $94.76 \%, 74.58 \%$ and $49.47 \%$ in the GP group, respectively, indicating no significant difference $(p>0.05)$. No statistical differences $(p>0.05)$ were also observed in DFS rates in 1 and 2 years between the DP group (76.5\% and $50.47 \%$, respectively) and GP group (77.8\% and $49.52 \%$, respectively).

\section{Discussion}

Surgery has been considered as the main treatment of stage II-III NSCLC, in which tumours are completely resected by expanding the range of patients with stage II-III NSCLC. At present, new adjuvant chemotherapy has been applied to patients diagnosed with stage III NSCLC to obtain definite efficacy. Cisplatin has been considered as one of the most important drugs to treat advanced NSCLC with a single-agent efficiency of $16 \%$ to $20 \%$ [9]. In recent years, the advent of several new chemotherapy drugs, including paclitaxel and gemcitabine combined with cisplatin for chemotherapy, has brought great progress in NSCLC treatment.

Docetaxel, classified as a broad-spectrum botanical antitumour and anticancer drug extracted and synthesised from needle-like leaves of yew trees, can form stable non-functional microbundles by strengthening microtubule polymerisation and inhibiting microtubule depolymerisation to inhibit mitosis of tumour cells [10]. Studies have shown that the combined treatment of docetaxel significantly increases the disease control rate of NSCLC and extends the progression-free survival of patients compared with docetaxel alone, but toxic responses are evidently enhanced [11]. In the case of combined docetaxel and platinum, the patients can develop dose-restricted bone marrow suppression as manifested by leucocytopenia [12]

Gemcitabine is a new type of synthetic pyrimidine nucleoside analogue with broad-spectrum antitumour activity [13]. Combined treatment of cisplatin and gemcitabine can increase their cytotoxic effects, thereby improving the efficiency of NSCLC treatment [14].

Liu et al. [15] compared the efficacy of three chemotherapies, including Navelbine (NVB), docetaxel or gemcitabine combined with cisplatin, in the treatment of advanced NSCLC by a prospective, open, randomised study, but the results did not show any significant difference. Some studies revealed that the efficiency of NVB, TAX or GEM combined with DDP in the treatment of advanced NSCLC ranges between $30.0 \%$ and $44.4 \%$ with a median survival ranging from 11 to 17 months; the survival rate ranges from $35.0 \%$ to $61.0 \%$, but these results did not have a statistical dif-
Table 1. Comparison of toxic response between two groups

\begin{tabular}{lcccc}
\hline & \multicolumn{2}{c}{ DP } & \multicolumn{2}{c}{ GP } \\
\hline Toxic response & I-II & III-IV & I-II & III-IV \\
\hline Leucocytopenia & 30 & 9 & 28 & 8 \\
\hline Thrombocytopenia & 22 & 11 & 17 & 3 \\
\hline Decrease of haemoglobin & 9 & 1 & 11 & 0 \\
\hline Hepatic dysfunction & 12 & 0 & 9 & 0 \\
\hline Nausea and vomiting & 22 & 0 & 20 & 0 \\
\hline Alopecia & 32 & 8 & 10 & 2 \\
\hline Allergy & 0 & 0 & 0 & 0 \\
\hline
\end{tabular}

ference [16-18]. Although GEM combined with platinum has not been used in adjuvant chemotherapy for stage III NSCLC yet, data from the treatment of advanced NSCLC and several clinical trials involving stage II NSCLC with GEM have indicated that the combined treatment of GEM with platinum is more efficacious than NVB therapy in terms of safety and efficacy of postoperative chemotherapy [19-21]. This treatment might become a standard scheme of postoperative chemotherapy for NSCLC.

In our research, a total of 92 patients with stage II-III NSCLC received postoperative chemotherapy of DP or GP. DFS rates in 1 and 2 years were $76.5 \%$ and $50.47 \%$ in the DP group and $77.8 \%$ and $49.52 \%$ in the GP group, respectively, but no significant difference was observed between the two groups ( $p>0.05$ ). Toxic responses, including bone marrow suppression and gastrointestinal reaction, were observed in the two groups. Gastrointestinal reaction was associated with cisplatin. The incidence of grade III-IV thrombocytopenia in the DP group was $24.4 \%$ and this incidence was higher than that of the GP group (6.38\%; $p<0.05)$. The incidence of alopecia in the DP group was also higher than that of the GP group.

The results showed a similar DFS rate and overall survival rate for DP and GP chemotherapy for the treatment of stage II-III NSCLC. However, the DP group exhibited higher incidence of grade III-IV thrombocytopenia and alopecia than the GP group. Therefore, we should select a specific treatment for each patient according to individual differences.

This study was supported by the Project of Science and Technology Bureau, Huzhou, Zhejiang (2010YSB07).

The authors declare no conflict of interest.

\section{References}

1. Boukovinas I, Kosmidis P. Treatment of non-small cell lung cancer patients with performance status2 (PS2). Lung Cancer 2009; 63: 10-5.

2. Le Chevaliera T. Adjuvant chemotherapy for resectable non-smallcell lung cancer: where is it going? Ann Oncol 2010; 21: 196-8. 
3. Spdsova I. The position of neoadjuvant chemotherapy in the treatment of non-small -cell lung carcinoma. Vnitr Lek 2007; 53: 715-23.

4. Gkiozos I, Charpidou A, Syrigos K. Developments in the treatment of non-small-cell lung cancer. Anticancer Res 2007; 27: 2823-7.

5. Barlési F, Doddoli C, Chetaille B, Torre JP, Giudicelli R, Thomas P, Kleisbauer JP, Fuentes P. Survival and postoperative complication in daily practice after neoadjuvant therapy in resectable stage IIIA-N2 non-small cell lung cancer. Interact Cardiovasc Thorac Surg 2003; 2: 558-62.

6. Le Chevalier T. In: Grunberg SM, (ed). Results of the randomized international adjuvant lung cancer trial (IALT) cisplatin-based chemotherapy (CT) versus no CT in 1867 patients with resected nonsmall cell lung cancer. Annual Meeting of the American Society of Clinical Oncology (ASCO) 2003 May 31-Jun.

7. Pisters KM, Evans WK, Azzoli CG, et al.; Cancer Care Ontario; American Society of Clinical Oncology. Cancer Care Ontario and Amer ican Society of Clinical Oncology adjuvant chemotherapy and adjuvant radiation therapy for stages I-IIIA resectable non small-cell lung cancer guideline. J Clin Oncol 2007; 25: 5506-18.

8. Trotti A, Bentzen SM. The need for adverse effects reporting standards in oncology clinical trials. J Clin Oncol 2004; 22: 19-22.

9. Stincheombe TE, Socinski MA. Considerations for second-line therapy of non-small cell lung cancer. Oncologist 2008; 13: 28-36.

10. Kim YH, Kim JS, Choi YH, et al. Phase II study of docetaxel and cisplatin combination chemotherapy in metastatic or unresectable localized non-small cell lung cancer. Int J Clin Oncol 2002; 7: 114-9.

11. Hom L, Visval A, Leighl NB. Docetaxel in non-small cell lung cancer: impact on quality of life and pharmacoeconomics. Drugs Aging 2007; 24: 411-28.

12. Charoentum C, Thongprasert S, Chewasakulyong B, Euathrongc hit J, Sorraritchingchai S, Munprakan S. Phase II study of cisplatin combined to irinotecan administered alternatingly with docetaxe in advanced non-small cell lung cancer. J Med Assoc Thai 2007; 90: 2506-12.

13. Di Maio M, Chiodini P, Georgoulias V, et al. Meta-analysis of single agent chemotherapy compared with combination chemotherapy as second-line treatment of advanced non-small cell lung cancer. J Clin Oncol 2009; 27: 1836-43.

14. Sandler AB, Nemunaitis J, Denham C, et al. Phase III trial of gemcitabine plus cisplatin versus cisplatin alone in patients with locally advanced or metastatic non-small-cell lung cancer. J Clin Oncol 2000; 18: 122-30.

15. Liu L, Wang XW, Li L, Zhang X, Zhang WD, Yu XJ. A randomized comparative trial of three combined regimens containing cisplatin for treatment of advanced non-small cell lung cancer. Ai Zheng 2006; 25: 990-4.

16. Le Chevalier T, Scagliottib G, Natalec R, et al. Efficacy of gemcitabine plus platinum chemotherapy compared with other platinum containing regimens in advanced non-small-cell lung cancer: a meta-analysis of survival outcomes. Lung Cancer 2005; 47 69-80.

17. Chen CH, Chang WC, Lin MC, Hsu JW, Chao TY, Tsao TC. Phase I study of paclitaxel (Genaxol) and cisplatin combination in treating Chinese patients with advanced non-small cell lung cancer (NSCLC). Lung Cancer 2002; 38: 91-6.

18. Bretti S, Manzin E, Loddo C, Berruti A, Bombaci S, Vellani G, Celano A. Gemcitabine plus cisplatin in the treatment of patients with advanced non-small cell lung cancer, a phase II study. Anticancer Res 2002; 2: 3039-43

19. Tibaldi C, Mazzoni E, Areabasso G, D’Incecco A, Antonuzzo A, Men coni $\mathrm{G}$, Falcone A. Cisplatin plus gemeitabine as adjuvant chemotherapy for radically resecte non-small-cell lung cancer: a pilot study. Clin Lung Cancer 2009; 10: 53-7.

20. Funai K, Takamochi K, Itaya T, et al. Feasibility study of adjuvant chemotherapy with gemeitabine and spit-dose cisplatin for completely resected non-small-cell lung cancer. Lung Cancer 2010; 68: 78-83.

21. Usami N, Yokoi K, Hasegawa Y, et al.; Central Japan Lung Study Group. Phase II study of carboplatin and gemeitabine as adjuvant chemotherapy in patients with completely resected on-small cell lung cancer: a report from the Central Japan Lung Study Group, CJLSG 0503 trial. Int J Clin Oncol 2010; 15: 583-7.

\section{Address for correspondence}

\section{Qiu-Qiang Chen}

Department of Thoracic Surgery

The directly affiliated hospital of Huzhou Teachers College, No. 158 Guangchanghou Road,

Huzhou, Zhejiang 313000, China

tel. +86-0572-2023728

fax +86-0572-2023728

e-mail: qiuqiangchencn@163.com

Submitted: 30.08 .2013

Accepted: $\quad 3.12 .2013$ 